\title{
Enhanced cercosporin production by co-culturing Cercospora sp. JNU001 with leaf-spot-disease-related endophytic bacteria
}

\author{
Tingan Zhou', Shiyu Yu', Yifan Hu', Yan Zhang ${ }^{2}$, Yuechen Song ${ }^{1}$, Jieyu Chu', Changmei Liu and Yijian Rao ${ }^{1 *}$ (i)
}

\begin{abstract}
Background: Owing to the excellent properties of photosensitization, cercosporin, one of naturally occurring perylenequinonoid pigments, has been widely used in photodynamic therapy, or as an antimicrobial agent and an organophotocatalyst. However, because of low efficiency of total chemical synthesis and low yield of current microbial fermentation, the limited production restricts its broad applications. Thus, the strategies to improve the production of cercosporin were highly desired. Besides traditional optimization methods, here we screened leafspot-disease-related endophytic bacteria to co-culture with our previous identified Cercospora sp. JNU001 to increase cercosporin production.
\end{abstract}

Results: Bacillus velezensis B04 and Lysinibacillus sp. B15 isolated from leaves with leaf spot diseases were found to facilitate cercosporin secretion into the broth and then enhance the production of cercosporin. After 4 days of co-culture, Bacillus velezensis B04 allowed to increase the production of cercosporin from $128.2 \mathrm{mg} / \mathrm{L}$ to $984.4 \mathrm{mg} / \mathrm{L}$, which was 7.68-fold of the previously reported one. Lysinibacillus sp. B15 could also enhance the production of cercosporin with a yield of $626.3 \mathrm{mg} / \mathrm{L}$, which was 4.89 -fold higher than the starting condition. More importantly, we found that bacteria B04 and B15 employed two different mechanisms to improve the production of cercosporin, in which B04 facilitated cercosporin secretion into the broth by loosening and damaging the hyphae surface of Cercospora sp. JNU001 while B15 could adsorb cercosporin to improve its secretion.

Conclusions: We here established a novel and effective co-culture method to improve the production of cercosporin by increasing its secretion ability from Cercospora sp. JNU001, allowing to develop more potential applications of cercosporin.

Keywords: Cercosporin, Co-culture, Endophytic bacteria, Secretion, Microbial fermentation

\footnotetext{
*Correspondence: raoyijian@jiangnan.edu.cn

1 Key Laboratory of Carbohydrate Chemistry and Biotechnology, Ministry of Education, School of Biotechnology, Jiangnan University, Wuxi 214122, People's Republic of China

Full list of author information is available at the end of the article
}

\begin{abstract}
Background
Cercosporin (CP), one of naturally occurring perylenequinonoid pigments (PQPs) with a characterized 3,10-dihydroxy-4,9-perylenequinone chromophore core structure (Fig. 1a), was first isolated in the mycelium of Cercospora kikuchii in 1957 and then was widely found in many pathogenic fungus Cercospora [1-3], which is a causal agent of leaf spot diseases (LSD) in a wide range of crops [4], including soybean [5], maize [6], and olive [7].
\end{abstract}

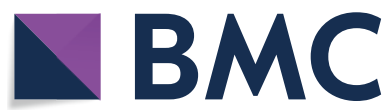

(c) The Author(s) 2021. This article is licensed under a Creative Commons Attribution 4.0 International License, which permits use, sharing, adaptation, distribution and reproduction in any medium or format, as long as you give appropriate credit to the original author(s) and the source, provide a link to the Creative Commons licence, and indicate if changes were made. The images or other third party material in this article are included in the article's Creative Commons licence, unless indicated otherwise in a credit line to the material. If material is not included in the article's Creative Commons licence and your intended use is not permitted by statutory regulation or exceeds the permitted use, you will need to obtain permission directly from the copyright holder. To view a copy of this licence, visit http://creativeco mmons.org/licenses/by/4.0/. The Creative Commons Public Domain Dedication waiver (http://creativecommons.org/publicdomain/ zero/1.0/) applies to the data made available in this article, unless otherwise stated in a credit line to the data. 
a<smiles>COc1c(C[C@H](C)O)c2c3c(C[C@H](C)O)c(OC)c4c(=O)c5c(O)cc6c-5c3-c3cc(O)c(c1=O)c(c3OCO6)c42</smiles>
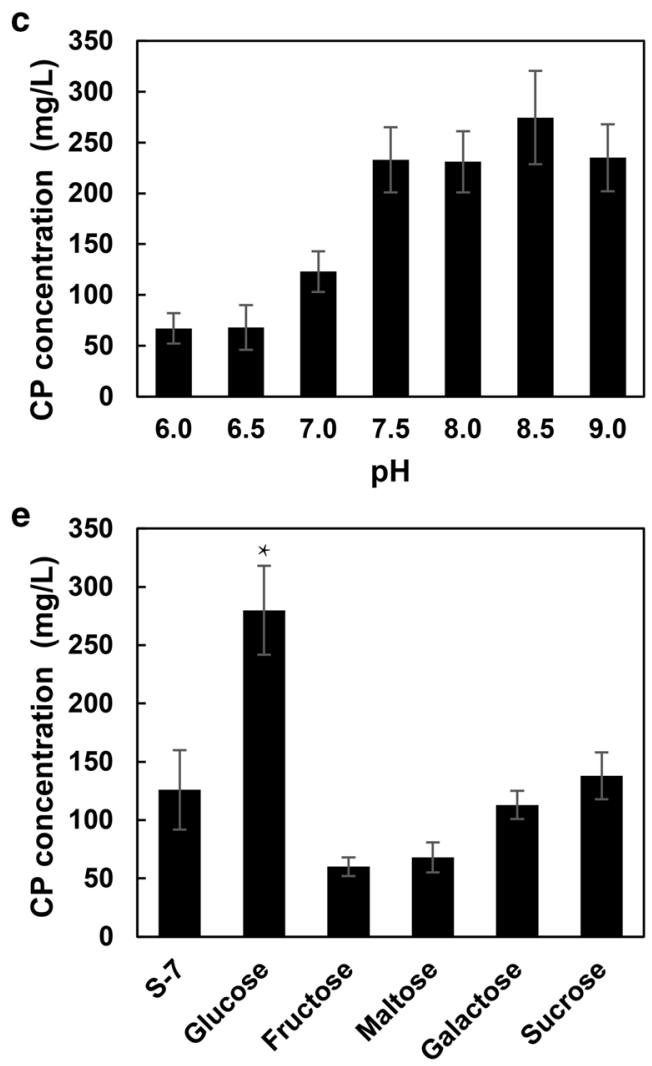

b
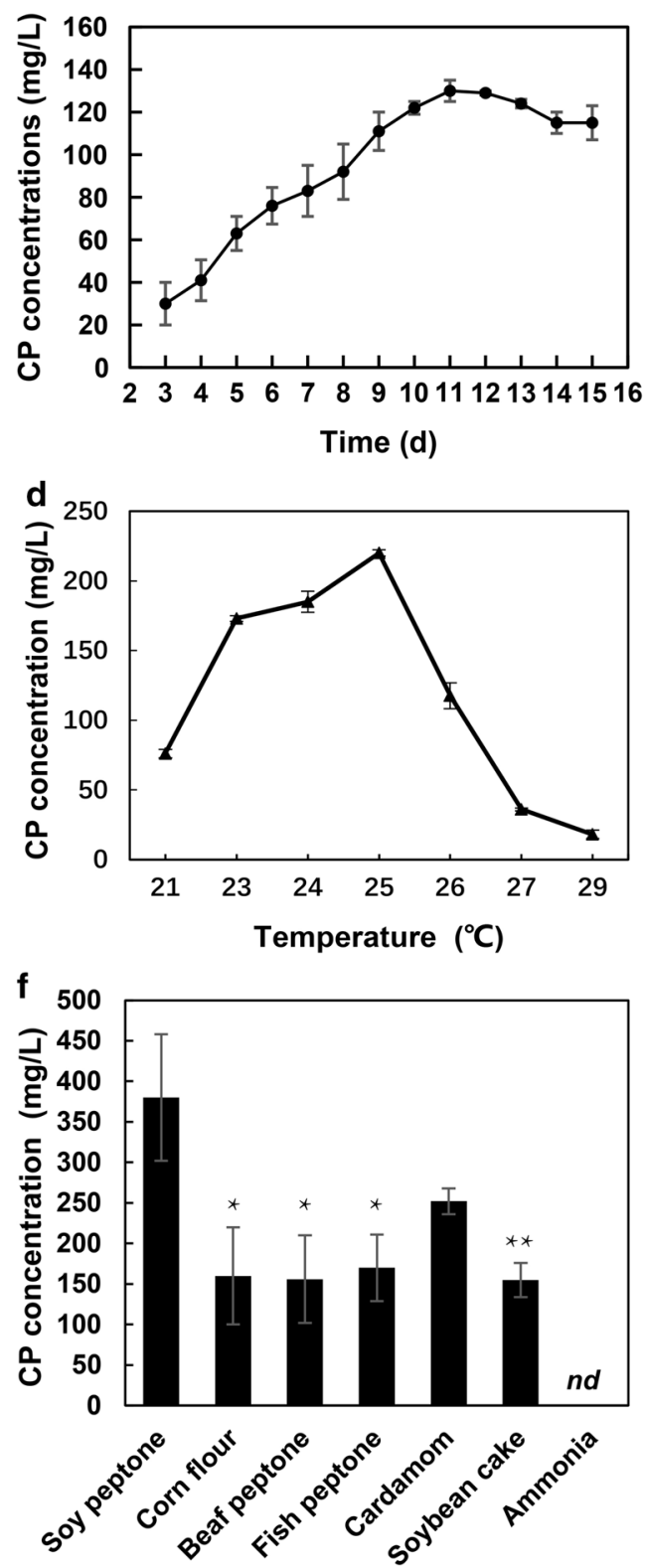

Fig. 1 Improvement of CP production by optimizing culture medium and culture conditions. a Molecular structure of CP. The characterized 3,10-dihydroxy-4,9-perylenequinone chromophore core structure was labelled in blue. $\mathbf{b}$ Influence of culture time on $\mathrm{CP}$ production. $\mathrm{d}=\mathrm{day}$. $\mathbf{c}$ pH optimization of S-7 medium. $\mathbf{d}$ Optimization of culture temperature. e Selection of carbon source in S-7 medium. $\mathbf{f}$ Screening of nitrogen source in S-7 medium (cardamom: cardamom powder, ammonia: ammonia sulfate, $n d$ : no detected). ${ }^{*} p<0.05$ and ${ }^{* *} p<0.01$ versus control (carbon/nitrogen source in traditional S-7 medium)

Owing to its excellent properties of photosensitization, it is widely investigated in the aspects of photophysics, photochemistry and photobiology [8-13], and has been used in photodynamic therapy and photophysical diagnosis, or as antimicrobial agents [14-16]. For instance, it has broad applications in the treatment of refractory skin diseases caused by certain fungi and cancer $[16,17]$. CP is also a potent inhibitor of protein kinase C (PKC) [18], which regulates numerous intracellular signal transduction, including cell differentiation, cell proliferation and inflammatory response, by controlling the function of other proteins through phosphorylation. More recently, we have developed CP as a new class of metal-free photocatalyst to catalyze a series of chemical transformations, 
including selective photo-oxidation, $\mathrm{C}-\mathrm{H}$ activation, $\mathrm{C}-\mathrm{N}$ coupling and $\mathrm{C}-\mathrm{S}$ coupling [19-23]. Meanwhile, it has also been utilized to fabricate a novel HARCP/ HAp photocatalyst (HexaAcetyl-Reductive Cercosporin/ Hydroxyapatite) after a simple structural modification, allowing to efficiently photoremove tetracycline in water pollution under natural sunlight [24]. Based on these applications of $\mathrm{CP}$ and potential industrial demands, a large quantity of $\mathrm{CP}$ is highly desired.

Currently, CP is mainly produced by total chemical synthesis and microbial fermentation. However, owing to its structural complexity (Fig. 1a), the total chemical synthesis of PQPs, including CP, needs more than twenty steps [18], which limits its practical application. Thus, the production of $\mathrm{CP}$ is mainly dependent on microbial fermentation by culturing fungus Cercospora sp. [22, 25-27]. So far, many previous studies have focused on increasing the productivity of $\mathrm{CP}$ by optimizing numerous fermentation factors, including medium, salts, buffers and ions, and delivered the maximum production of $75.59 \mathrm{mg} / \mathrm{L}$ after 31 days culture of a Cercospora strain from symptomatic leaves of water hyacinth [26]. However, both of solid-state and liquid fermentation are currently not able to produce stable and high-yield of $\mathrm{CP}$ in a large scale within a reasonable culture time. Additionally, its productivity highly varies from different sources of Cercospora sp. [2]. Thus, the improvement of $\mathrm{CP}$ production through different strategies by a promising Cercospora strain is still awaiting to be explored.

Recently, we identified a new CP-producing strain Cercospora sp. JNU001, which was isolated from bark of Taxus chinensis, with the ability to produce CP at the yield of $128.2 \mathrm{mg} / \mathrm{L}$ when it was cultivated under continuous light illumination with S-7 medium after 11 days [22], which is substantially higher than other previous studies, even within a shortened culture time [26]. Therefore, herein we attempted to improve the production of CP using Cercospora sp. JNU001 strain. We initiated the improvement of $\mathrm{CP}$ production of Cercospora sp. JNU001 by typical medium optimization and culture condition optimization, and then employed the co-cultivation method, a powerful ecologically driven approach to increase the production of specific metabolites or to produce some new substances by mimicking natural situations [28-33]. To our delight, besides the enhanced production of CP by typical medium optimization and culture condition optimization, the production of $\mathrm{CP}$ could be further improved by co-culturing with two new identified LSD endophytic bacteria Bacillus velezensis B04 and Lysinibacillus sp. B15. The yields of CP were $984.4 \mathrm{mg} / \mathrm{L}$ (Bacillus velezensis B04) and 626.3 $\mathrm{mg} / \mathrm{L}$ (Lysinibacillus sp. B15), which were 7.68-fold and 4.89-fold higher than the starting condition, respectively.
Furthermore, we found that these two bacteria applied different mechanisms to improve the production of $\mathrm{CP}$.

\section{Results and discussion \\ Determination and optimization of liquid fermentation conditions}

Considering the limited production of $\mathrm{CP}$ on PDA plate [22], S-7 culture medium was firstly chosen as the basic medium to optimize the liquid fermentation conditions $[19,22,34]$, including culture time, medium $\mathrm{pH}$, temperature, carbon source and nitrogen source (Fig. 1b-f). It was found that the production of $\mathrm{CP}$ was dramatically increased from $128.2 \mathrm{mg} / \mathrm{L}$ to $467.8 \mathrm{mg} / \mathrm{L}$ when Cercospora sp. JNU001 was cultured at $25{ }^{\circ} \mathrm{C}$ with the modified S-7 medium (initial $\mathrm{pH}=8.5$ ) (Fig. $1 \mathrm{~b}-\mathrm{d}$ ), in which glucose was used as carbon source and soy peptone as nitrogen source (Fig. 1e, f), for 11 days under continuous light illumination. The total amount of CP was 6.19-fold and 3.65-fold higher than the previously reported condition [26], and our original condition [35], respectively. For the Cercospora sp. JNU001 strain, its production ability reached the maximum at 11 days and then part of $\mathrm{CP}$ was degraded when the culture time was increased (Fig. 1b), which is consistent with previous studies [35]. Surprisingly, the production of CP was almost inhibited when the culture temperature was set at more than $27^{\circ} \mathrm{C}$ (Fig. 1d). Moreover, no CP was produced when inorganic ammonia was used as nitrogen source (Fig. 1f). Thus, we obtained the highest productivity of $\mathrm{CP}$ after typical optimizations through liquid fermentation, allowing us to further improve $\mathrm{CP}$ production by co-culture strategy.

\section{Screening of leaf-spot-disease-related bacteria}

As co-cultivation often enhances the production of metabolites by mimicking natural situations $[29,36]$, we began with screening the endophytic bacteria community related to LSD to mimic the phenomenon caused by pathogen Cercospora sp., and then co-cultured each of them with Cercospora sp. JNU001 to further increase $\mathrm{CP}$ production. After extensive purification, a total of 16 pure bacteria were isolated from the LSD related leaves (Additional file 1: Table S1). Next, each of them was cocultured with Cercospora sp. JNU001 to investigate their effect on the production of $\mathrm{CP}$ under the above optimized conditions. It was found that most of them had a negative effect on its production from 92.72 to $10.84 \%$ (Additional file 1: Table S1). The B10 strain even caused the death of Cercospora sp. JNU001 (Additional file 1: Table S1). To our delight, B04 and B15 strains had a positive effect to increase the production of $\mathrm{CP}$ (Fig. 2 and Additional file 1: Table S1). Furthermore, ${ }^{1} \mathrm{H}$ NMR analysis showed that the product purified from co-cultivation was still CP (Additional file 1: Fig. S1). Thus, these results 


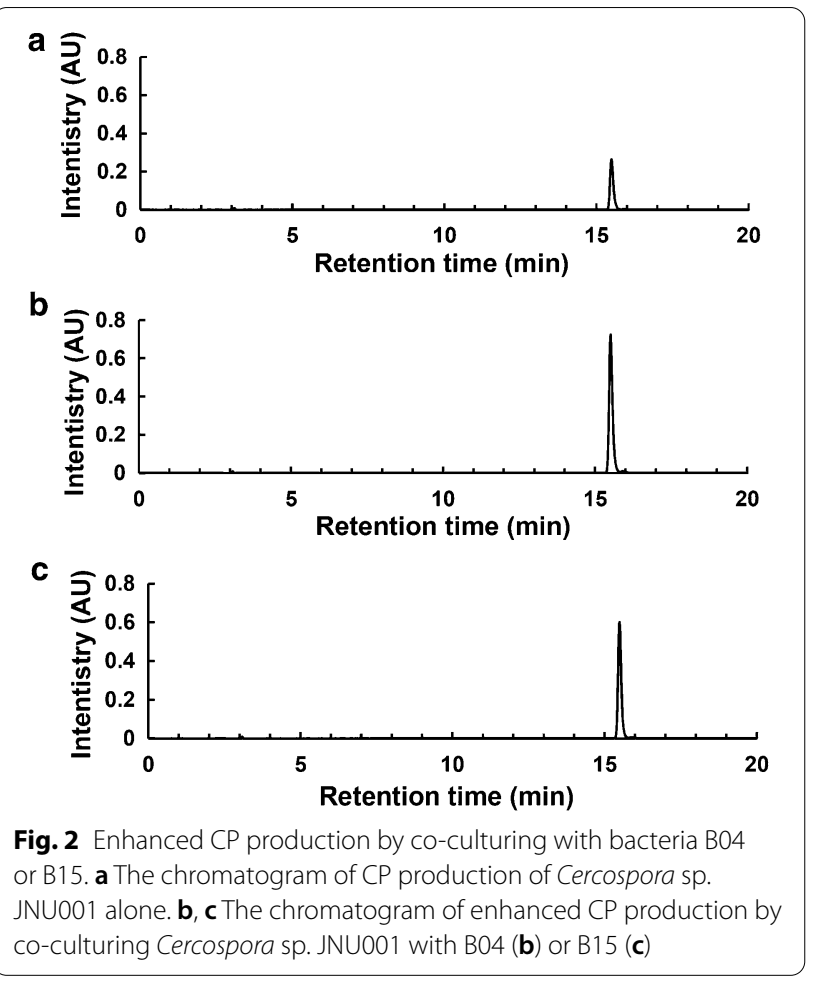

allowed us to further characterize B04 and B15 strains and then investigated how they improved the production of CP.

\section{Identification and characterization of B04 and B15 strains}

It showed that the B04 colony appeared round, rough and white in color (Fig. 3a), while the B15 colony appeared round, smooth, small and white in color (Fig. 3b), suggesting that different molecular mechanisms might be applied by B04 and B15 to increase the production of CP. Based on the analysis of $16 \mathrm{~S}$ rDNA nucleotide sequences (GenBank accession number MW418038.1 for B04, MW418069.1 for B15, respectively), the phylogenetic tree for B04 or B15 strain was established through the alignment and cladistics analysis of homologous nucleotide sequence (Fig. 3c, d). B04 strain and B15 strain belonged to Bacillus velezensis and Lysinibacillus sp., respectively. Compared to Bacillus velezensis CBMB205 (GenBank accession number, NR_116240.1), B04 strain has a similarity of $99.57 \%$, which was then designated as Bacillus velezensis B04 (Fig. 3c). B15 strain shows a similarity of 99.43\% with Lysinibacillus macroides LMG18474 (GenBank accession number, NR_114920.1) (Fig. 3d), and then was named as Lysinibacillus sp. B15.

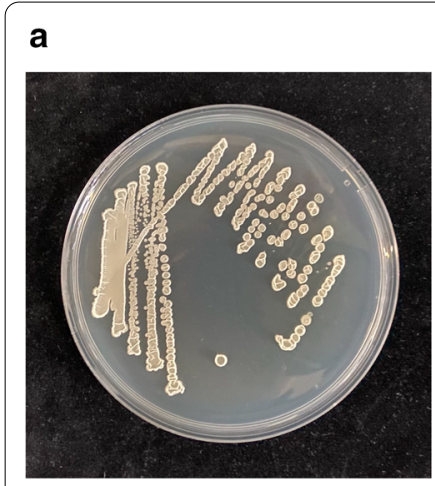

C

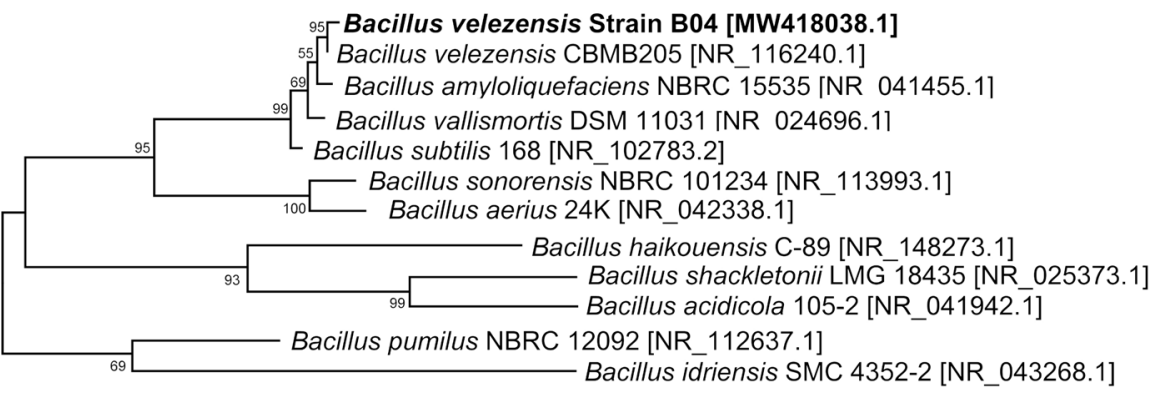

b

d

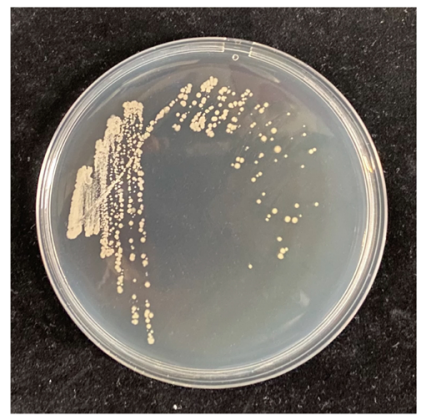

44__Lysinibacillus macroides LMG 18474 [NR_114920.1]

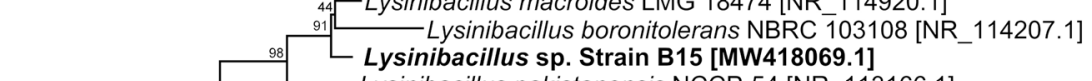

Lysinibacillus pakistanensis NCCP-54 [NR_113166.1]

Lysinibacillus fusiformis DSM 2898 [NR_042072.1]

[ Lysinibacillus mangiferihumi M-GX18 [NR 118146.1]

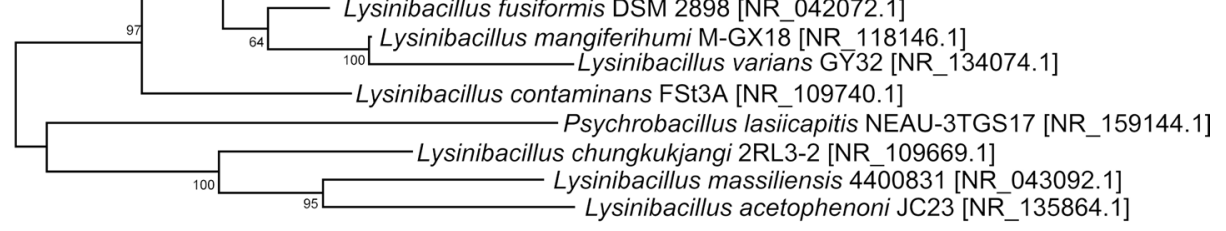

Fig. 3 Identification and characterization of two isolated bacteria B04 and B15. a, b Microscopic appearance of culture colony of B04 strain and B15 strain on LB plate after $24 \mathrm{~h}$. c The phylogenetic tree of B04 strain and its relationship with other Bacillus species. $\mathbf{d}$ The phylogenetic tree of B15 strain and its relationship with other Lysinibacillus species 


\section{Optimization of co-culture conditions to enhance CP production}

Next, we optimized co-culture conditions by adding different amounts of B04 or B15 to Cercospora sp. JNU001 pre-cultures, which initially grew overnight and then was diluted to different concentrations with the modified S-7 medium (Fig. 4a, b). It showed that B04 could greatly enhance the production of CP at different concentrations compared with the condition without co-culturing with B04 in modified S-7 medium, and the $\mathrm{CP}$ production reached a maximum of 984.4 $\mathrm{mg} / \mathrm{L}$ when B04 was added at the final concentration of $0.20 \mathrm{OD}_{600}$ (Fig. 4a), which was 2.67 -fold and 7.68 -fold higher than the one in modified S-7 medium and the original condition [35], respectively. However, its production decreased when more B04 was added (Fig. 4a). Interestingly, the production of $\mathrm{CP}$ only increased when the B15 strain was added around $0.20 \mathrm{OD}_{600}$, in
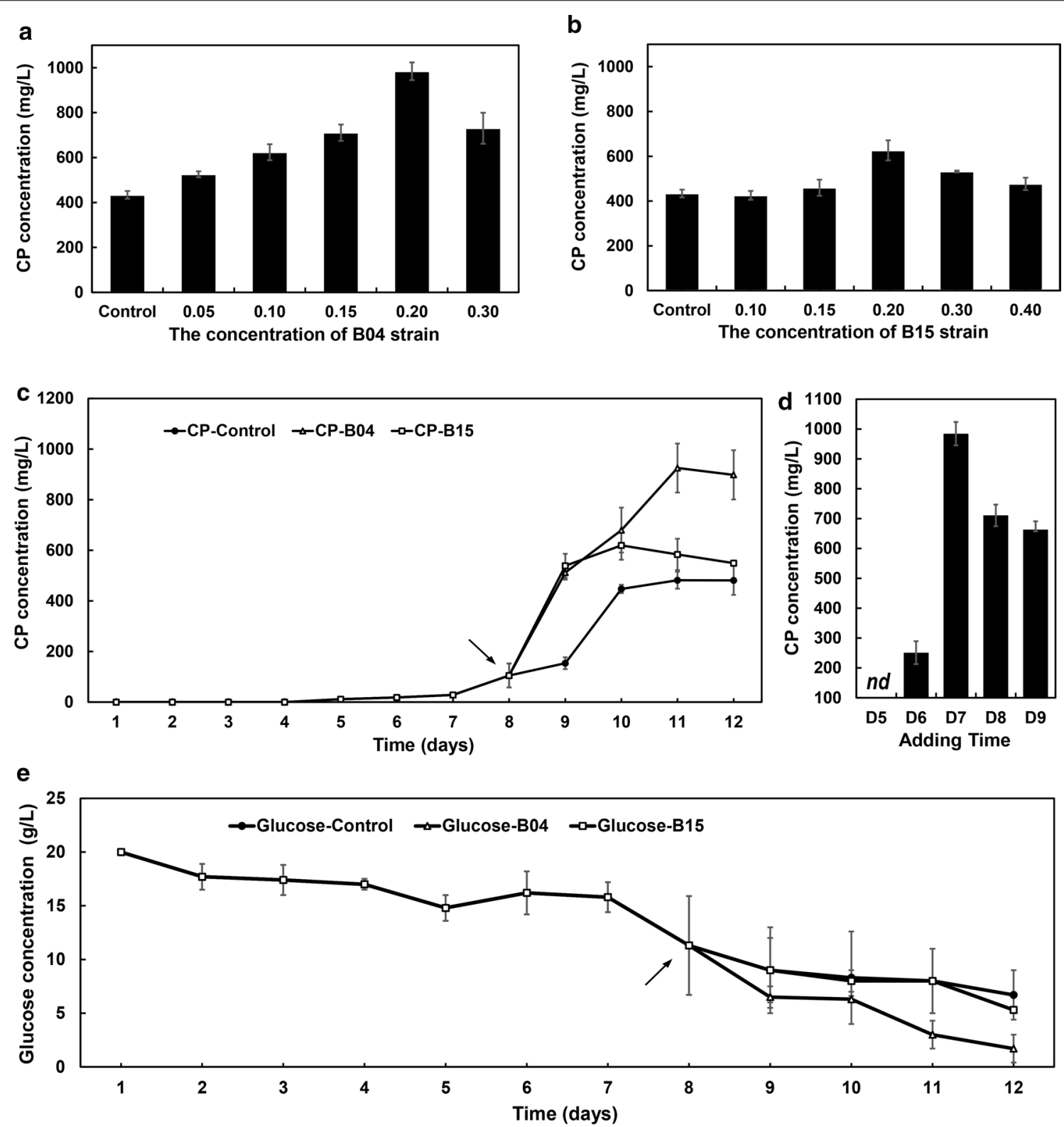

Fig. 4 Effect of CP production by co-culturing B04 or B15 strain with Cercospora sp. JNU001. a, b Effect of the concentration of B04 or B15 strain on CP production. c. Effect of culture time on CP production when Cercospora sp. JNU001 was co-cultured with B04 or B15. $\mathbf{d}$ Effect of the adding time of B04 on CP production. e The glucose consumption of Cercospora sp. JNU001 grew in the modified S-7 medium and under co-culture with B04 or B15 strain condition. The arrows indicate the beginning of significant differences caused by co-culture 
which the highest production of $\mathrm{CP}$ was achieved at $626.3 \mathrm{mg} / \mathrm{L}$, which was 1.33 -fold of that in modified S-7 medium (Fig. 4b). No significant increase was observed when less or more B15 strain was used (Fig. 4b).

To better understand the effect of co-culturing Cercospora sp. JNU001 with B04 or B15 strain, their timecourse of $\mathrm{CP}$ production were analyzed. It showed that the amount of $\mathrm{CP}$ was very low at the beginning phase (Fig. 4c), and then started to secrete more CP after day 7. Under the control strain, the production of $\mathrm{CP}$ reached the maximum at day 11 (Fig. 4c), similar with the unmodified S-7 medium (Fig. 1b). Considering the growing speed of bacteria and Cercospora sp. JNU001 and the antimicrobial activity of $\mathrm{CP}$, the appropriate time to add B04 or B15 was around day 7, in which Cercospora sp. JNU001 began to produce CP and then could use its antimicrobial activity to introduce the growth pressure and competition for bacteria. To verify the hypothesis, the effect of the adding time of B04 (day $5,6,7,8$ and 9) was analyzed. Surprisingly, no CP was detected when B04 was added at day 5 (Fig. 4d). The production of $\mathrm{CP}$ was impaired when B04 was added at day 6. Interestingly, although the amount of $\mathrm{CP}$ was enhanced when the B04 strain was added at day 8 and 9 , the production of $\mathrm{CP}$ was significantly impaired when compared with the condition at day 7 (Fig. 4d), illustrating that the optimal time to add B04 was day 7. Moreover, the maximum of $\mathrm{CP}$ production also happened at day 11 (Fig. 4c). After day 11, Cercospora sp. JNU001 appeared to autolyze and had a negative effect on CP production (Additional file 1: Fig. S2). Similarly, the same phenomenon was observed for the strain B15 (Fig. 4c).

To further support the aforementioned conclusions, we also investigated the glucose utilization by measuring the remaining glucose concentration during the time-course of fermentation (Fig. 4e). It clearly showed that the glucose utilization was greatly increased after day 7 no matter with or without co-culturing with B04 or B15 (Fig. 4e), which was well correlated with the production of $\mathrm{CP}$ (Fig. 4c). Interestingly, the glucose utilization was similar under control condition and B15 co-culture condition, but more glucose was consumed under B04 co-culture condition after day 7 , probably owing to the requirement of more energy to synthesize $\mathrm{CP}$ as it delivered much more $\mathrm{CP}$ than the other two conditions. Moreover, the remaining glucose was very limited after day 12 under B04 co-culture condition, which could explain the autolysis of Cercospora sp. JNU001 (Additional file 1: Fig. S2).

\section{Effect of live B04 and B15 on fungal growth and cercosporin secretion}

To understand molecular mechanisms that improved the production of CP by B04 or B15 strain, we next performed in vitro fungal-bacterial confrontation bioassays (Fig. 5a, b) [37, 38]. It showed that B04 and B15 strains resulted in different phenomena (Fig. 5c). Surprisingly, Cercospora sp. JNU001 was unable to cross the boundary of the B04 strain (Fig. 5c(i-iv)), but it clearly induced the secretion of $\mathrm{CP}$ as it was well distributed outside of the boundary of Cercospora sp. JNU001 with the disappearance of the red ring of $\mathrm{CP}$ (Fig. $5 \mathrm{c}(\mathrm{iv})$ ), in which the growth of both of B04 and Cercospora sp. JNU001 was somehow inhibited (Fig. 5c(iii, iv)). On the contrary, Cercospora sp. JNU001 obviously crossed over the boundary of B15 (Fig. 5c(v-viii)). Moreover, the red ring of CP still existed and B15 bacteria also became red once they got contacted with Cercospora sp. JNU001 (Fig. 5c(viii)), suggesting that $\mathrm{B} 15$ had the ability to adsorb $\mathrm{CP}$ to stimulate its secretion and then enhance its production. To verify this hypothesis, we then investigated whether B15 could emit the red fluorescence from $\mathrm{CP}$ after co-culturing with Cercospora sp. JNU001. As expected, the B15 strain alone did not show any fluorescence, but became red after coculturing with Cercospora sp. JNU001 (Additional file 1: Fig. S3), confirming that CP could be adsorbed by B15 strain to facilitate its production, which was supported by other study that the adsorption ability could enhance the production of metabolites [39]. In fact, this result was also supported by our own investigation, in which the production of CP was improved with the yield of 787.9 $\mathrm{mg} / \mathrm{L}$ when XAD-16 resins, the macroporous materials with excellent potential adsorption capacity, were used as a substitute to replace B15 strain (Additional file 1: Fig. S4).

Next, we investigated whether the above phenomena would also happen in the liquid fermentation condition. It was found that there was no obvious difference of dry biomass between the control strain and B04 co-culture condition (Fig. 6a), suggesting that there was no influence on fungal growth under B04 co-culture conditions. However, the dry biomass of Cercospora sp. JNU001 was slightly decreased when it was co-cultured with B15. Interestingly, the amount of $\mathrm{CP}$ extracted from the dry biomass of $\mathrm{B} 15$ co-culture was similar with the control strain, but slightly decreased in the B04 co-culture system (Fig. 6b), probably due to the excellent secretion ability of CP induced by B04 (Fig. 5c(iv), 6c). As the proportion of $\mathrm{CP}$ secreted into the culture broth was higher than the one extracted from dry biomass in both B04 and B15 co-culture conditions, which mainly contributed to the production of $\mathrm{CP}$ (Fig. $6 \mathrm{~d}$ ), it was suggested that both of B04 and B15 could facilitate CP secretion to improve 


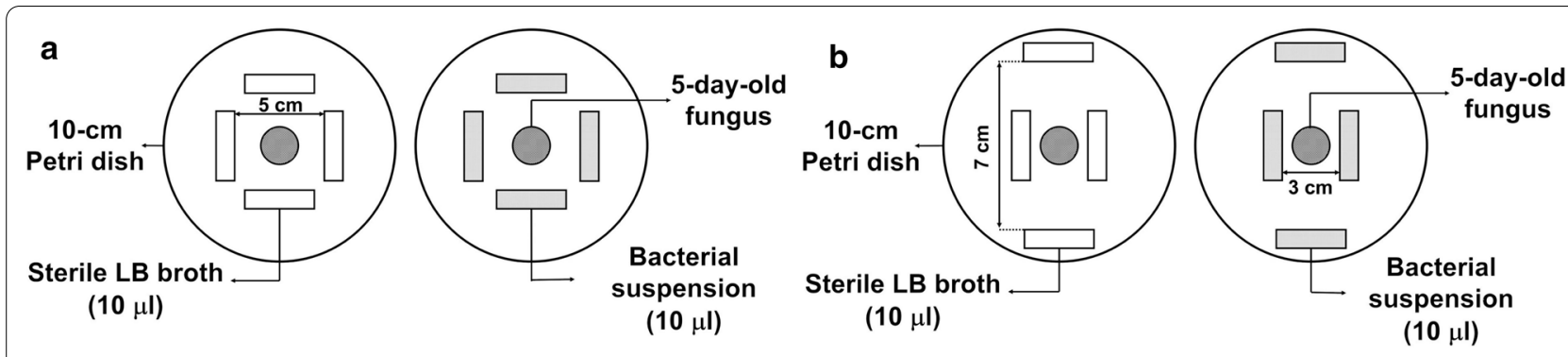

C

\section{Co-culture}

Top
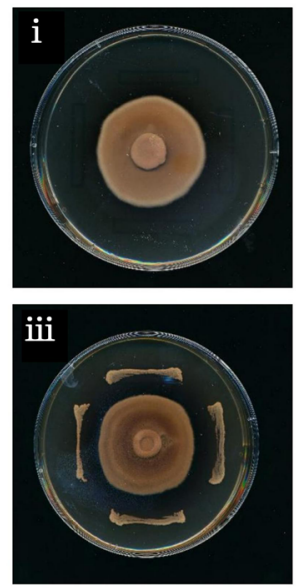

Reverse
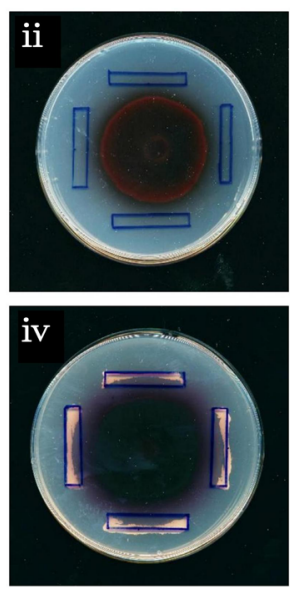

Top
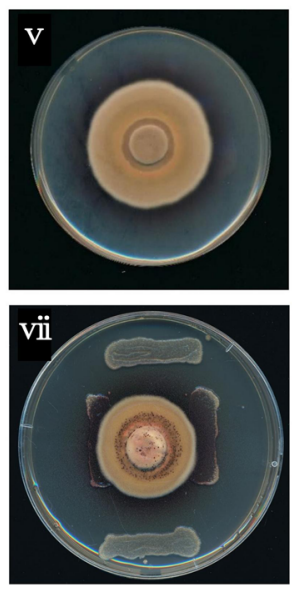

Reverse
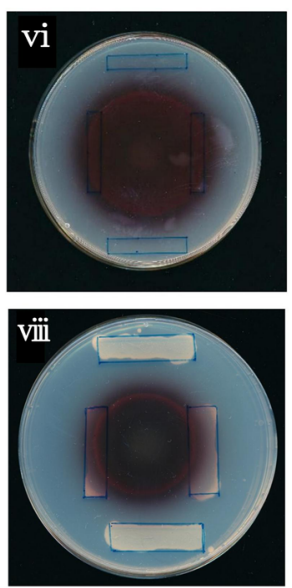

Fig. 5 In vitro confrontation bioassays between bacteria and Cercospora sp. JNU001. a Schematic diagram of in vitro confrontation bioassay between B04 and Cercospora sp. JNU001. b Schematic diagram of in vitro confrontation bioassay between B15 and Cercospora sp. JNU001. c Effects of B04 (i-iv) or B15 (v-viii) on Cercospora sp. JNU001

its production. Furthermore, the secretion ability of $\mathrm{CP}$ induced by the B04 strain was much better than the B15 strain (Fig. 6c), implying that B04 and B15 might employ two different mechanisms to improve the production of CP.

\section{Morphological observation of co-culture samples}

To further support the above conclusion that two different mechanisms were applied by B04 and B15 to increase the production of $\mathrm{CP}$ through enhancing its secretion ability, field emission scanning electron microscope (FESEM) was employed to investigate the morphology of co-culture samples (Fig. 7), which was derived from the optimized liquid fermentation. It showed that the bacteria B04 were attached on the fungal hyphae surface and seemed to have the capacity to loosen it (Fig. $7 d-f)$, even to damage the fungal hyphae (Fig. 7e, f), which was very tight in the original Cercospora sp. JNU001 strain (Fig. 7b, c). To further support these phenomena, the Congo red differential medium with glucan, which is the main component of fungal cytoderm and can be degraded by glucanase [40-42], was employed to determine whether it would be degraded by B04. Indeed, the glucan around the B04 strain, but not B15 strain, was able to be degraded (Additional file 1: Fig. S5), indicating that B04 probably have an ability to secrete glucanase to loosen and damage the fungal hyphae [43], which could facilitate cercosporin secretion and then resulted in the improvement of CP production. To further support this notion, the commercial glucanase was directly added to Cercospora sp. JNU001 culture at day 7 , and the CP production was indeed increased with the yield of $719.7 \mathrm{mg} / \mathrm{L}$ (Additional file 1: Fig. S6). Furthermore, it showed that bacteria B04 were somehow shrunk and became unhealthy when compared with the untreated ones (Fig. 7a, d-f).

However, as for the B15 co-culture condition, no obvious appearance change of Cercospora sp. JNU001 was observed, and only a few bacteria B15 had physical attachment on the surface of hyphae (Additional file 1: Fig. S7), in which the shape of B15 bacteria was also deformed like the bacteria B04.

Together with the result that $\mathrm{CP}$ was adsorbed and inserted into B15 bacteria (Additional file 1: Fig. S3), we concluded that bacteria B04 and B15 employed different 

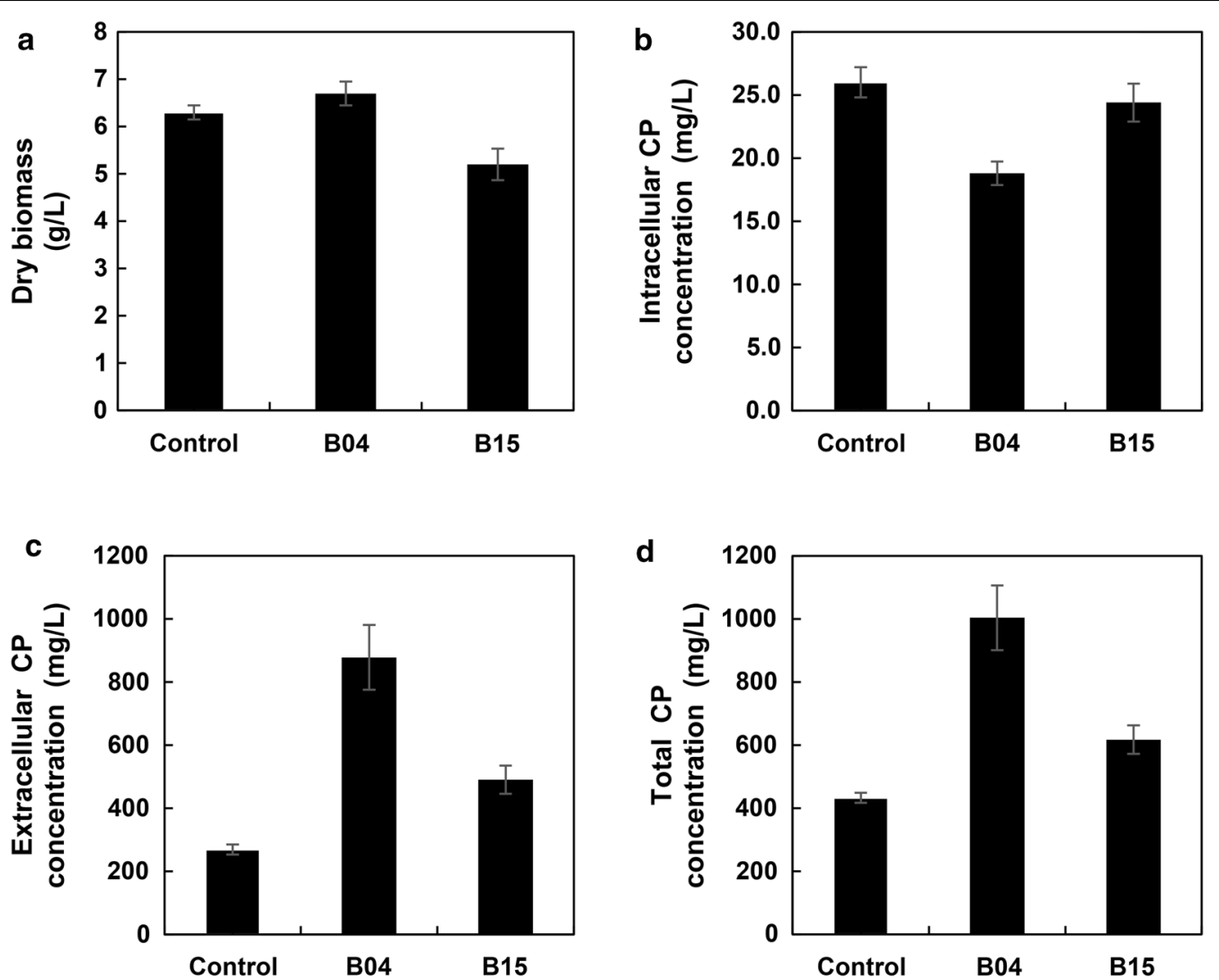

Fig. 6 Effects of B04 and B15 on fungal growth and CP secretion. a Dry fungal biomass of Cercospora sp. JNU001 without or with bacteria. $\mathbf{b}$ Intracellular CP production of Cercospora sp. JNU001 extracted from a. c Extracellular CP production purified from culture broth after co-culture without or with bacteria. $\mathbf{d}$ Total CP production, which was calculated by intracellular CP production (b) and extracellular CP production (c)
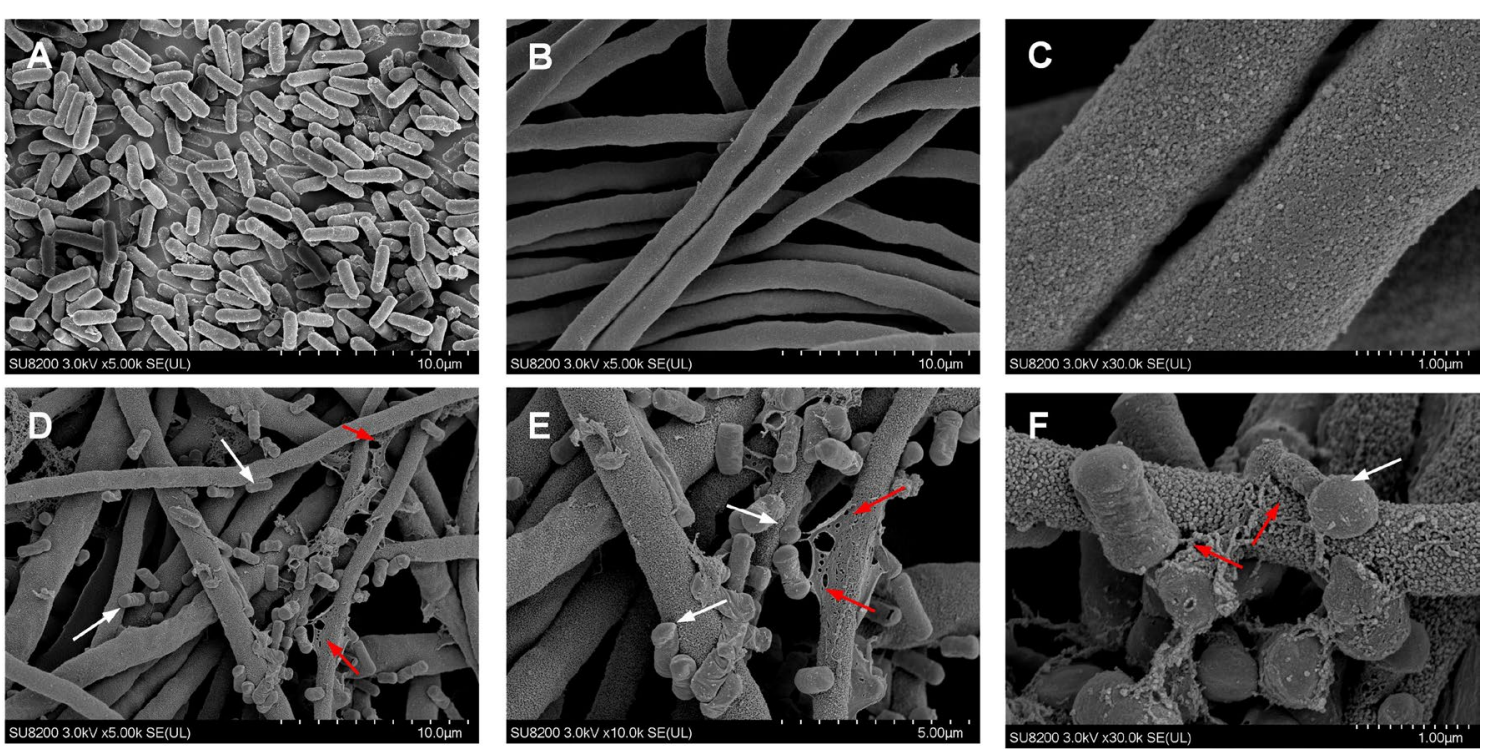

Fig. 7 FESEM observation of co-culture Cercospora sp. JNU001 with B04. a B04 samples. b, c Cercospora sp. JNU001 samples. d-f Co-culture samples of B04 and Cercospora sp. JNU001. White arrows indicate bacteria B04, and red arrows indicate the damage of fungal hyphae. Scale bar was indicated 
mechanisms to enhance the production of CP, in which B04 could loosen and damage the hyphae of Cercospora sp. JNU001 to facilitate CP secretion while B15 had an ability to adsorb CP to improve its secretion. Surprisingly, the production of $\mathrm{CP}$ was not further improved but inhibited with the yield of $280.4 \pm 2.9 \mathrm{mg} / \mathrm{L}$ when both of B04 and B15 were co-cultured with Cercospora sp. JNU001.

\section{Conclusions}

To improve the production of $\mathrm{CP}$, besides traditional optimization methods, including optimization of culture medium and culture conditions, the co-culture method was also employed in this study. After extensive screening and purification, two new identified bacteria Bacillus velezensis B04 and Lysinibacillus sp. B15 further enhanced the production of $\mathrm{CP}$ by separately co-culturing with fungus Cercospora sp. JNU001 with the yield of $984.4 \mathrm{mg} / \mathrm{L}$ and $626.3 \mathrm{mg} / \mathrm{L}$, which were 7.68-fold and 4.89-fold of that in the starting condition, respectively. Moreover, two different mechanisms were found to increase the production of $\mathrm{CP}$ by these two bacteria. Bacillus velezensis B04 had the ability to loosen or damage the surface of hyphae and then to improve the secretion ability of CP, while Lysinibacillus sp. B15 could adsorb and accumulate $\mathrm{CP}$ to increase $\mathrm{CP}$ production. Thus, we here provided a novel effective co-culture method to enhance CP production of Cercospora sp., which allows to develop more applications of $\mathrm{CP}$.

\section{Materials and methods CP-producing fungal strain and culture conditions} $\mathrm{CP}$ was produced by an endophytic fungus Cercospora sp. JNU001, which was isolated from the tree bark of Taxus chinensis in Lishui, Zhejiang, China and reserved in China Center for Type Culture Collection (CCTCC 2017842). The strain was stored on modified S-7 solid medium (glucose: $20 \mathrm{~g} / \mathrm{L}$, sodium acetate: $1 \mathrm{~g} / \mathrm{L}$, soy peptone: $2 \mathrm{~g} / \mathrm{L}$, phenylalanine: $5 \mathrm{mg} / \mathrm{L}$; sodium benzoate: $100 \mathrm{mg} / \mathrm{L}, 1 \mathrm{M} \mathrm{KH}_{2} \mathrm{PO}_{4}$ buffer: $1 \mathrm{~mL}$, biotin: $1 \mathrm{mg} / \mathrm{L}$, $\mathrm{Ca}\left(\mathrm{NO}_{3}\right)_{2}: 6.5 \mathrm{mg} / \mathrm{L}$, pyridoxal: $1 \mathrm{mg} / \mathrm{L}$, calcium pantothenate: $1 \mathrm{mg} / \mathrm{L}$, thiamine: $1 \mathrm{mg} / \mathrm{L}, \mathrm{MnCl}_{2}: 5 \mathrm{mg} / \mathrm{L}, \mathrm{FeCl}_{3}: 2$ $\mathrm{mg} / \mathrm{L}, \mathrm{Cu}\left(\mathrm{NO}_{3}\right)_{2}: 1 \mathrm{mg} / \mathrm{L}, \mathrm{MgSO}_{4}: 3.6 \mathrm{mg} / \mathrm{L}, \mathrm{ZnSO}_{4}: 2.5$ $\mathrm{mg} / \mathrm{L}$, agar powder: $15 \mathrm{~g} / \mathrm{L}$ ) at $4{ }^{\circ} \mathrm{C}$, or in cryotubes with glycerol $(20 \%)$ at $-80{ }^{\circ} \mathrm{C}$.

The traditional S-7 medium before optimization was shown below: Glucose: $1 \mathrm{~g} / \mathrm{L}$, fructose: $2 \mathrm{~g} / \mathrm{L}$, saccharose: $6 \mathrm{~g} / \mathrm{L}$ sodium acetate: $1 \mathrm{~g} / \mathrm{L}$, soy peptone: $2 \mathrm{~g} / \mathrm{L}$, phenylalanine: $5 \mathrm{mg} / \mathrm{L}$; sodium benzoate: $100 \mathrm{mg} / \mathrm{L}, 1 \mathrm{M} \mathrm{KH}_{2} \mathrm{PO}_{4}$ buffer: $1 \mathrm{~mL}$, biotin: $1 \mathrm{mg} / \mathrm{L}, \mathrm{Ca}\left(\mathrm{NO}_{3}\right)_{2}: 6.5 \mathrm{mg} / \mathrm{L}$, pyridoxal: $1 \mathrm{mg} / \mathrm{L}$, calcium pantothenate: $1 \mathrm{mg} / \mathrm{L}$, thiamine: 1 $\mathrm{mg} / \mathrm{L}, \mathrm{MnCl}_{2}: 5 \mathrm{mg} / \mathrm{L}, \mathrm{FeCl}_{3}: 2 \mathrm{mg} / \mathrm{L}, \mathrm{Cu}\left(\mathrm{NO}_{3}\right)_{2}: 1 \mathrm{mg} / \mathrm{L}$, $\mathrm{MgSO}_{4}: 3.6 \mathrm{mg} / \mathrm{L}, \mathrm{ZnSO}_{4}: 2.5 \mathrm{mg} / \mathrm{L}$, agar powder: $15 \mathrm{~g} / \mathrm{L}$.

Cercospora sp. JNU001 was inoculated into $500 \mathrm{~mL}$ flasks with $100 \mathrm{~mL}$ modified S-7 liquid medium and then cultured at $25^{\circ} \mathrm{C}$ in a shaker (ZQZY-AF8, Zhichu, China) with $135 \mathrm{rpm}$ upon continuous light illumination (flasks were fixed $16.67 \pm 3.00 \mathrm{~cm}$ away from a $15 \mathrm{~W}$ compact fluorescent lamp (CFL)).

\section{Separation and purification of CP}

$50 \mathrm{~mL}$ dichloromethane (DCM) was added to the fermentation broth after 11 days and then flasks were put back to the shaker at $135 \mathrm{rpm}$ for $36 \mathrm{~h}$ to ensure the complete extraction of $\mathrm{CP}$. This procedure was repeated twice. The organic phase containing CP was collected and DCM was evaporated (RV8, IKA, Germany) to obtain the raw $\mathrm{CP}$, which was then dissolved in methanol and purified by a Sephadex column LH-20.

\section{Quantitative determination of CP production and glucose consumption}

To rapidly determine the content of $\mathrm{CP}$, the raw $\mathrm{CP}$ was analyzed by HPLC (2695, Waters, America) using an Aglient column $\left(5 \mu \mathrm{m}, \mathrm{C}_{18}, 100 \AA\right.$, $\left.4.6 \times 250 \mathrm{~mm}\right)$ with the characteristic adsorption wavelength of $\mathrm{CP}$ at 472 $\mathrm{nm}$. The purified CP was used as a reference. Then, the concentration of $\mathrm{CP}$ of each sample could be calculated. To study the time-course of CP production, $2 \mathrm{~mL}$ of culture broth was taken out at each day and extracted three times by $2 \mathrm{~mL}$ DCM. The extracted fraction was collected and DCM was evaporated. The raw materials with CP was dissolved in $200 \mu \mathrm{L}$ methanol and loaded into HPLC. The amount of CP was detected by HPLC. The gradient was as follows: $0-20 \mathrm{~min}, 20-85 \%$ Solvent A; $20-23 \mathrm{~min}$, 85\% Solvent A; $23-27$ min, $85-20 \%$ Solvent A; 27-30 min, 20\% Solvent A. Solvent A: HPLC grade acetonitrile containing $0.5 \%$ formic acid; Solvent B: HPLC grade $\mathrm{H}_{2} \mathrm{O}$ containing $0.5 \%$ formic acid. The solvent flow rate was $1.0 \mathrm{~mL} / \mathrm{min}$. The column was pre-equilibrated for $10 \mathrm{~min}$ between injections.

To study the glucose consumption, $100 \mu \mathrm{L}$ culture broth was taken out from the flask each day and diluted with double distilled water $\left(\mathrm{dd}_{2} \mathrm{O}\right)$. The diluted broth was centrifuged at 12,000 rpm to remove bacterium and fungal mycelia, and then injected to SBA-40E Biosensor Analyzer, which was made by Institute of Biology, Shandong Academy of Science, to measure the glucose concentration.

\section{Isolation and identification of leaf-spot-disease-related endophytic bacteria}

Leaves with LSD was collected from August to October in Wuxi, Jiangsu, China. All leaf samples were immediately 
stored in sealed bags on ice and then sterilized as the following procedures [44]. The fresh leaves were washed by tap water for $1-2 \mathrm{~h}$ and cut into small pieces $(2-3 \mathrm{~cm}$ long and $0.5 \mathrm{~cm}$ wide), which were then rinsed by sterilized water for 3 times, dipped in $75 \%$ ethyl alcohol for $1 \mathrm{~min}$ and again rinsed by sterilized water for 3 times. Next, some new wounds of leaf pieces were made by the sterilized scalpel to contact LB agar plate (tryptone: $10 \mathrm{~g} / \mathrm{L}$, yeast extra: $5 \mathrm{~g} / \mathrm{L}$, sodium chloride: $10 \mathrm{~g} / \mathrm{L}$, agar powder: $15 \mathrm{~g} / \mathrm{L}, \mathrm{pH}: 7.0-7.2)$ after drying with sterilized filter tissue paper and then cultured at $37^{\circ} \mathrm{C}$ without light for $48 \mathrm{~h}$. The single colony was obtained by streak plate method, and then inoculated into LB medium to cultivate without light at $37^{\circ} \mathrm{C}$ in a shaker at $200 \mathrm{rpm}$ for 24 h. Each of purified bacteria was stored in cryovial tubes with glycerol $(25 \%)$ at $-80^{\circ} \mathrm{C}$.

To characterize each of purified bacteria, the bacterial general primers, 27F (5'-AGAGTTTGATCATGGCTC AG- $3^{\prime}$ ) and 1492R (5'-TACGGCTACCTTG TTACGA CTT-3') were used to amplify the $16 \mathrm{~S}$ rDNA of each of them. The PCR reaction was performed in a final volume of $50 \mu \mathrm{L}: 1 \mu \mathrm{L}$ bacteria culture medium containing DNA, $25 \mu \mathrm{L}$ of $2 \times$ Premix, $1 \mu \mathrm{L}$ of $27 \mathrm{~F}(5 \mathrm{nmol} / \mathrm{L})$ and $1 \mu \mathrm{L}$ of $1492 \mathrm{R}(5 \mathrm{nmol} / \mathrm{L}), 22 \mu \mathrm{L}$ double distilled water $\left(\mathrm{ddH}_{2} \mathrm{O}\right)$. The amplified PCR product was purified and sequenced by GENEWIZ Inc. (Suzhou, China). The obtained sequences were uploaded to GenBank in NCBI database and the cladogram was constructed by neighbor-joining method (1000-replicate bootstrap) in MEGA-X (version 10.1.8) after homologous comparisons with the existed bacterial sequences in NCBI. The scale bar of the cladogram indicated the number of base substitutions per site.

\section{Establishment of co-culture conditions}

To improve the production of CP, two small pieces (5 $\mathrm{mm} \times 5 \mathrm{~mm}$ ) of Cercospora sp. JNU001 from the modified S-7 agar plate were firstly inoculated into $100 \mathrm{~mL}$ modified S-7 liquid medium at $25^{\circ} \mathrm{C}$ on a rotating shaker at $135 \mathrm{rpm}$ for 7 days. At day 6, the single colony of each of two isolated bacteria B04 and B15 were inoculated into $50 \mathrm{~mL} \mathrm{LB}$ medium and cultured at $37{ }^{\circ} \mathrm{C}$ on a rotating shaker at $200 \mathrm{rpm}$ overnight. At day 7, the customized number of bacteria cells was harvested, centrifuged and resuspended by modified S-7 liquid medium, and then added into pre-culture Cercospora sp. JNU001. The coculture samples grew for another $3-5$ days at $25^{\circ} \mathrm{C}$ on a rotating shaker at $135 \mathrm{rpm}$ with continuous light illumination (flasks were fixed $16.67 \pm 3.00 \mathrm{~cm}$ away from a 15 W compact fluorescent lamp (CFL)). After that, $50 \mathrm{~mL}$ DCM was added into culture broth to extract $\mathrm{CP}$ using the aforementioned method. The dry biomass was measured immediately after treatment in vacuum freeze dryer
(FreeZone 6 Plus, LABCONCO, America) for 3 days. Similarly, $50 \mathrm{~mL}$ DCM was also used to extract CP from dry biomass of Cercospora sp. JNU001. The content of CP was detected by HPLC.

\section{In vitro fungal-bacterial confrontation bioassay}

Based on the method reported by Wang et al. [38], different kinds of confrontation bioassays (in vitro) were conducted between Cercospora sp. JNU001 and each of two isolated bacteria. Firstly, a small piece $(5 \mathrm{~mm} \times 5 \mathrm{~mm})$ of marginal mycelium of Cercospora sp. JNU001 with agar was dug out and reset in the center of a $10 \mathrm{~cm}$ modified S-7 agar plate and let it grow for 5 days. Next, the single colony of different bacteria was inoculated in LB medium at $37{ }^{\circ} \mathrm{C}$ on a rotating shaker at $200 \mathrm{rpm}$ for $24 \mathrm{~h}$ on day 5 , respectively. As it is shown in Fig. 5, bacterial suspension $(10 \mu \mathrm{L})$ was streaked in four parallel rectangular areas (approximately $3 \mathrm{~cm} \times 0.5 \mathrm{~cm}$ ) and cultured for another 10 days. The morphology was observed.

\section{Morphological observation}

The physical attachment between Cercospora sp. JNU001 and B04 or B15 was observed by FESEM (SU-8220, Hitachi, Japan). The samples were collected at day 11 (3 days after co-culturing). Meanwhile, pure bacteria and fungus were also observed as a control. To study whether B15 had an ability to adsorb CP, B15 samples were collected from in vitro fungal-bacterial confrontation assays when Cercospora sp. JNU001 partially reached the edge of the rectangular area of B15, and then dissolved by double distilled water. $10 \mu \mathrm{L}$ was dropping onto a glass slide and measured by a fluorescence microscope (CKX53, OLYMPUS, Japan) as CP has the capacity to emit the fluorescence. Meanwhile, B15 samples far from Cercospora sp. JNU001 on the in vitro fungal-bacterial confrontation assays were also collected and used as a control.

\section{In-situ adsorption of XAD-16 resins on CP}

To investigate whether XAD-16 resins had the similar effect like B15 strain on enhanced production of CP, XAD-16 resins $(30 \mathrm{~g} / \mathrm{L})$, which was pretreated as other reports [45], were added to Cercospora sp. JNU001 cultures at day 7. CP was then extracted by DCM, resolved in methanol and analyzed by HPLC.

\section{Congo red stain}

To study whether B04 or B15 had an ability to secret glucanase to damage the fungal hyphae, Congo red agar plate $\left(0.05 \% \mathrm{~K}_{2} \mathrm{HPO}_{4}, 0.05 \% \mathrm{MgSO}_{4}, 0.05 \% \mathrm{NaCl}, 0.2 \%\right.$ $\left(\mathrm{NH}_{4}\right)_{2} \mathrm{SO}_{4}, 0.5 \%$ glucan, $0.2 \%$ Congo red and $1.5 \%$ agar) was prepared. Next, B04 or B15 on LB plate was dug out, transferred onto the center of glucan Congo red agar 
plate, and let it grow for another 4-5 days. The transparent zone was scanned.

\section{Supplementary Information}

The online version contains supplementary material available at https://doi. org/10.1186/s12934-021-01587-2.

Additional file 1: Table S1. 16 bacteria isolated from leaves with leaf spot diseases and their effects on CP production by co-cultivation. Figure $\mathbf{S 1}$. ${ }^{1} \mathrm{H}$ NMR of CP structure. Figure S2. Autolysis of Cercospora sp. JNU001. Figure S3. Fluorescence microscope observation of B15 after culturing with Cercospora sp. JNU001. Figure S4. Effect of XAD-16 resins on its CP production. Figure S5. Analysis of the ability of B04 and B15 to degrade glucan. Figure S6. Influence of glucanase on CP production. Figure S7. FESEM observation of Cercospora sp. JNU001 with B15.

\section{Authors' contributions}

$T Z, S Y, Y H, Y S$ and JC performed the experiments. TZ, YZ and $C L$ analyzed the data. YR and TZ wrote the manuscript. YR designed and supervised this project. All authors read and approved the final manuscript.

\section{Funding}

This study is supported by the National Key R\&D Program of China (2018YFA0901700), the Natural Science Foundation of Jiangsu Province (BK20202002) and Jiangnan University National Undergraduate Innovative Training Programme (202010295045)

\section{Availability of data and materials}

All data generated or analyzed during this study are included in this published article and its Additional file.

\section{Declarations}

\section{Ethics approval and consent to participate} Not applicable.

\section{Competing interests}

The authors declare that they have no competing interests.

\section{Author details}

${ }^{1}$ Key Laboratory of Carbohydrate Chemistry and Biotechnology, Ministry of Education, School of Biotechnology, Jiangnan University, Wuxi 214122, People's Republic of China. ${ }^{2}$ School of Pharmaceutical Science, Jiangnan University, Wuxi 214122, People's Republic of China.

Received: 18 February 2021 Accepted: 28 April 2021

Published online: 15 May 2021

\section{References}

1. Daub ME. Cercosporin, a photosensitizing toxin from Cercospora species. Phytopathol. 1982;72:370-4.

2. Fajola AO. Cercosporin, a phytotoxin from Cercospora spp. Physiol Plant Pathol. 1978;13:157-64.

3. Kuyama S, Tamura T. Cercosporin. A pigment of Cercosporina Kikuchii Matsumoto et Tomoyasu. I. Cultivation of fungus, isolation and purification of pigment. J Am Chem Soc. 1957:79:5725-6.

4. Chupp TC. Notes on some Cercosporae of India. Mycologia. 1948;40:352-62.

5. Reznikov S, De Lisi V, Claps P, González V, Devani MR, Castagnaro AP, Ploper LD. Evaluation of the efficacy and application timing of different fungicides for management of soybean foliar diseases in northwestern Argentina. Crop Prot. 2019;124:

6. Crous PW, Groenewald JZ, Groenewald M, Caldwell P, Braun U. Species of Cercospora associated with grey leaf spot of maize. Stud Mycol. 2006:55:189-97.
7. Avila A, Groenewald JZ, Trapero A, Crous PW. Characterisation and epitypification of Pseudocercospora cladosporioides, the causal organism of Cercospora leaf spot of olives. Mycol Res. 2005;109:881-8.

8. Mulrooney CA, O'Brien EM, Morgan BJ, Kozlowski MC. Perylenequinones: isolation, synthesis, and biological activity. Eur J Org Chem. 2012;2012:3887-904.

9. Daub ME. Resistance of fungi to the photosensitizing toxin, cercosporin Phytopathol. 1987;77:1515-20.

10. Daub ME, Briggs SP. Changes in tobacco cell membrane composition and structure caused by cercosporin. Plant Physiol. 1983;71:763-6.

11. Daub ME, Ehrenshaft M. The photoactivated Cercospora toxin cercosporin: contributions to plant disease and fundamental Biology. Annu Rev Phytopathol. 2000;38:461-90.

12. Diwu Z, Lown JW. Photosensitization with anticancer agents: 15. Perylenequinonoid pigments as potential photodynamic therapeutic agents: formation of semiquinone radicals and reactive oxygen species on illumination. J Photochem Photobiol B. 1993;18:131-43.

13. Guedes RC, Eriksson LA. Photophysics, photochemistry, and reactivity: molecular aspects of perylenequinone reactions. Photochem Photobiol Sci. 2007:6:1089-96.

14. Dobrowolski D, Foote C. Cercosporin, a singlet oxygen generator. Angew Chem Int Ed. 2010;22:720-1.

15. Kumarihamy M, Khan SI, Jacob M, Tekwani BL, Duke SO, Ferreira D, Nanayakkara NP. Antiprotozoal and antimicrobial compounds from the plant pathogen Septoria pistaciarum. J Nat Prod. 2012;75:883-9.

16. Mastrangelopoulou M, Grigalavicius M, Berg K, Ménard M, Theodossiou TA. Cytotoxic and photocytotoxic effects of cercosporin on human tumor cell lines. Photochem Photobiol Sci. 2019;95:1.

17. Grigalavicius M, Mastrangelopoulou M, Arous D, Juzeniene A, Ménard M, Skarpen E, Berg K, Theodossiou TA. Photodynamic efficacy of cercosporin in 3D tumor cell cultures. Photochem Photobiol. 2020;96:699-707.

18. Morgan BJ, Dey S, Johnson SW, Kozlowski MC. Design, synthesis, and investigation of protein kinase $C$ inhibitors: total syntheses of $(+)$-calphostin $D_{1}(+)$-phleichrome, cercosporin, and new photoactive perylenequinones. J Am Chem Soc. 2009;131:9413-25.

19. Li J, Bao W, Tang Z, Guo B, Zhang S, Liu H, Huang S, Zhang Y, Rao Y. Cercosporin-bioinspired selective photooxidation reactions under mild conditions. Green Chem. 2019;21:6073-81.

20. Li J, Bao W, Zhang Y, Rao Y. Metal-free fercosporin-photocatalyzed C-S coupling for the selective synthesis of aryl sulfides under mild conditions. Eur J Org Chem. 2019;2019:7175-8.

21. Tang Z, Li J, Lin F, Bao W, Zhang S, Guo B, Huang S, Zhang Y, Rao Y. Cercosporin-bioinspired photoreductive activation of aryl halides under mild conditions. J Catal. 2019;380:1-8.

22. Zhang S, Tang Z, Bao W, Li J, Guo B, Huang S, Zhang Y, Rao Y. Perylenequinonoid-catalyzed photoredox activation for the direct arylation of (het) arenes with sunlight. Org Biomol Chem. 2019;17:4364-9.

23. Zhang Y, Cao Y, Lu L, Zhang S, Rao Y. Perylenequinonoid-catalyzed $[4+1]$ - and [4+2]-annulations of azoalkenes: photocatalytic access to 1,2,3-thiadiazole/1,4,5,6-tetrahydropyridazine derivatives. J Org Chem. 2019;84:7711-21.

24. Lu L, Zhang Y, Yuan Z, Xu J, Li M, Wu Y, Wang L, Huang S, Rao Y. Easily fabricated HARCP/HAp photocatalyst for efficient and fast removal of tetracycline under natural sunlight. Chem Eng J. 2021;412:

25. Djebali N, Gaamour N, Badri M, Aouani ME. Optimizing growth and conidia production of Cercospora medicaginis. Phytopathol Mediterr. 2010;49:267-72.

26. Jiménez MM, Bahena SM, Espinoza C. Trigos n: isolation, characterization, and production of red pigment from Cercospora piaropi a biocontrol agent for Waterhyacinth. Mycopathologia. 2010;169:309-14.

27. Lynch FJ, Geoghegan MJ. Production of cercosporin by Cercospora species. Trans Br Mycol Soc. 1977;69:496-8.

28. Dong J, Zhang X, Baol J, Xu X, Qi S. Secondary metabolites of the coculture of Aspergillus sp. SCSGAF 0076 and Bacillus sp. MNMCCE 001. Acta Microbiol Sin. 2014;54:1289-95.

29. Jennifer W, Hassan HM, Marcel J, Rainer E, Rateb ME. Dual induction of new microbial secondary metabolites by fungal bacterial co-cultivation. Front Microbiol. 2017:8:1284.

30. Ma YJ, Zheng LP, Wang JW. Bacteria associated with Shiraia fruiting bodies influence fungal production of hypocrellin A. Front Microbiol. 2023;2019:10. 
31. Xu D, Wang L, Du C. Progress in microbial co-culture-A review. Acta Microbiol Sin. 2015;55:1089-96.

32. Zhou L, Miao Z, Xu D, Yang Z, Du C. Comparative analysis of co-culture and pure culture of antibacterial metabolites of Brevibacillus laterosporus BL-21 and Bacillus subtilis HNDF2. Chin Agric Sci Bull. 2017;33(022):118-25.

33. Zhu Y, Liu J, Liu J, Du G, Zhou J, Chen J. A high throughput method to screen companion bacterium for 2-keto-l-gulonic acid biosynthesis by co-culturing Ketogulonicigenium vulgare. Process Biochem. 2012;47:1428-32.

34. Stierle A, Strobel G, Stierle D. Taxol and taxane production by Taxomyces andreanae, an endophytic fungus of Pacific yew. Science. 1993:260:214-6.

35. Tang Z, Bao W, Guo B, Rao Y. Screening, identification and fermentation optimization of a cercosporin-producing strain. Sciencepap Online. 2019;01:14.

36. Marmann A, Aly A, Lin W, Wang B, Proksch P. Co-cultivation-A powerful emerging tool for enhancing the chemical diversity of microorganisms. Mar Drugs. 2014;12:1043-65.

37. Ma YJ, Zheng LP, Wang JW. Inducing perylenequinone production from a bambusicolous fungus Shiraia sp. S9 through co-culture with a fruiting body-associated bacterium Pseudomonas fulva SB1. Microb Cell Fact. 2019;18:121.

38. Wang XM, Yang HW, Ren CG, Zheng HL, Dai CC. Consequences of antagonistic interactions between endophytic fungus and bacterium on plant growth and defense responses in Atractylodes lancea. J BASIC MICROB. 2015;55(5):659-70.

39. Mei J, Min H, Lü Z. Enhanced biotransformation of L-phenylalanine to 2-phenylethanol using an in situ product adsorption technique. Process Biochem. 2009;44:886-90.
40. Kasana RC, Salwan R, Dhar H, Dutt S, Gulati A. A rapid and easy method for the detection of microbial cellulases on agar plates using Gram's iodine. Curr Microbiol. 2008:57:503-7.

41. Semedo MC, Karmali A, Fonseca L. A high throughput colorimetric assay of $\beta-1,3-d$-glucans by Congo red dye. J Microbiol Methods. 2015;109:140-8.

42. Wood PJ, Erfle JD, Teather RM. Use of complex formation between Congo Red and polysaccharides in detection and assay of polysaccharide hydrolases. Methods Enzymol. 1988;160:59-74.

43. XuT, Zhu T, Li S. $\beta$-1,3-1,4-glucanase gene from Bacillus velezensis ZJ20 exerts antifungal effect on plant pathogenic fungi. World J Microbiol Biotechnol. 2016;32:26.

44. Song PY, Lan QY, Lu ZY. Identification and phylogenetic analysis on endophytic bacteria isolated from Taxus chinensis var mairei. Biotechnol. 2012;22:61-5

45. Zheng Y, Wang D, Xu P, Shen W, Zhang Y. In-situ adsorption kinetics of macroporous resin on natamycin. Food Mach. 2019;12:42-6.

\section{Publisher's Note}

Springer Nature remains neutral with regard to jurisdictional claims in published maps and institutional affiliations.
Ready to submit your research? Choose BMC and benefit from:

- fast, convenient online submission

- thorough peer review by experienced researchers in your field

- rapid publication on acceptance

- support for research data, including large and complex data types

- gold Open Access which fosters wider collaboration and increased citations

- maximum visibility for your research: over 100M website views per year

At BMC, research is always in progress.

Learn more biomedcentral.com/submissions 